\title{
Miniaturized laser Doppler vibrometers integrated on silicon-on-insulator with thermo-optic serrodyne optical frequency shifter
}

\author{
Yanlu Li, Steven Verstuyft, Gunay Yurtsever, Shahram Keyvaninia, Gunther Roelkens, Dries Van Thourhout, Roel Baets \\ Photonics Research Group, INTEC Department, Ghent-University - IMEC \\ Center for Nano - and Biophotonics (NB-Photonics), Ghent University \\ Sint-Pietersnieuwstraat 41, 9000 Ghent, Belgium \\ Email: Yanlu.Li@intec.ugent.be
}

\begin{abstract}
A miniaturized heterodyne laser Doppler vibrometer integrated on silicon-on-insulator is reported and compared with a commercial LDV. The optical frequency shift was realized by applying the serrodyne method on a thermo-optic phase modulator.
\end{abstract}

\section{INTRODUCTION}

Laser Doppler vibrometers (LDVs) are devices that are used to measure the instantaneous velocity or displacement of vibrating surfaces using the Doppler effect. Thanks to their ability to make non-contact vibration measurements, LDVs are used in a variety of applications. Many efforts have been made in the miniaturization of LDVs, in order to reduce the fabrication cost, increase the mobility, and improve the interferometer's stability [1-3]. It also makes it easier to realize multi-point LDVs with miniaturized single point LDVs. In this paper, we propose to use the silicon-oninsulator (SOI) photonic integrated circuit (PIC) technologies for the miniaturization of LDVs. The SOI technology is CMOS compatible, which makes it possible to dramatically reduce the manufacturing cost for high production volumes. Thanks to the small waveguide bend on this platform [4], the footprint of a single LDV circuit can be scaled down to less than $1 \mathrm{~mm}^{2}$.

The proposed LDVs use the optical heterodyne detection technique. In this technique, coherent light with frequency $f_{0}$ is first split into two paths: a measurement path and a reference path. The measurement light is focused on the vibrating surface by using a lens system. A portion of the reflection is recaptured into the interferometer again. The electric field strength of the reflected measurement signal is expressed as $\alpha(t) A \cdot \exp \left[j\left(2 \pi f_{0} t+\theta(t)\right)\right]$, where $A$ is the electric amplitude of the measurement signal before sent out of the chip, $\alpha(t)$ is the loss of the reflected measurement signal, and $\theta(t)$ is the Doppler phase change due to the vibration of the surface of interest. The reference light undergoes a frequency shift $f_{O F S}$ with the help of an optical frequency shifter (OFS), and is expressed as $B \cdot \exp \left[j\left(2 \pi\left(f_{0}+f_{O F S}\right) t\right)\right]$. The reference and measurement lights are recombined in an optical combiner, and received by a photodetector. Since the reference light is optical frequency shifted, a beat is formed in the mixed signal and it can be sensed by the photo-detector. The photocurrent is expressed as

$$
\begin{aligned}
I(t) & \propto\left|\alpha(t) A \cdot e^{j\left[2 \pi f_{0} t+\theta(t)\right]}+B \cdot e^{j 2 \pi\left(f_{0}+f_{O F S}\right) t}\right| \\
& =\alpha^{2}(t) A^{2}+B^{2}+2 \alpha(t) A B \cos \left[2 \pi f_{O F S} t-\theta(t)\right] .
\end{aligned}
$$

With this photo-current signal, the instantaneous phase change $\theta(t)$ and frequency shift $f_{D}(t)=1 / 2 \pi \cdot \frac{d \theta(t)}{d t}$ of the vibrating surface can be retrieved using a frequency demodulator. The instantaneous velocity $v(t)$ of the vibrating surface is obtained using the linear relation between the instantaneous frequency shift and the vibration velocity

$$
v(t)=\frac{2 f_{D}(t)}{f_{0}} c .
$$

In this equation, $c$ is the speed of the light in the medium between the LDV and vibrating surface.

The common method for generating an optical frequency shift is to use the acoustic optic effect. The most common acoustic optic modulators (AOMs) are Bragg cells. However, since the acoustic optic effect is absent from an unstrained crystalline silicon, it is hard to realize an AOM on SOI. The optical frequency shifter reported in this paper is a thermooptic (TO) serrodyne frequency shifter, which can be used to generate a frequency shift up to several kilo-hertz [5]. Serrodyne modulation means that the optical phase of the light is modulated by a sawtooth signal in order to generate a frequency shift.

The PIC design and the measurement setup will be described in section II. In section III, the measurement results of the on-chip LDV will be compared with a commercial LDV.

\section{DESIGN AND MEASUREMENT SETUP}

In this paper, we report a demonstration of a Michelsoninterferometer (MI) type LDV circuit on SOI substrate. The design is shown schematically in Figure 1. In this design, light is sent into the on-chip interferometer via a standard grating coupler. The measurement light is sent out of the chip via a tilted grating coupler which was designed to suppress 


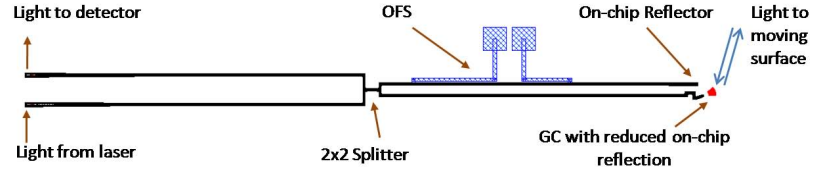

Figure 1. Schematic show of a Michelson type on-chip LDV with thermooptic based serrodyne optical frequency shifter (OFS). In the figure, $G C$ means grating coupler.

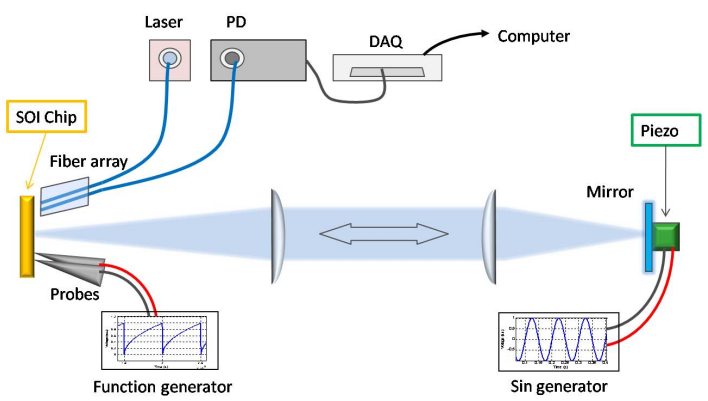

Figure 2. The setup used to measure mirror vibrations with on-chip LDVs. In the figure, $P D$ stands for photo-detector, and $D A Q$ for data acquisition card.

on-chip reflections [6]. The reflected measurement light is coupled back to the chip via the same tilted grating coupler. An on-chip reflector is used to reflect the reference light. The measurement and reference lights are recombined in the $2 \times 23 \mathrm{~dB}$ multimode-interferomter (MMI) and sent to an external photo-detector via a second standard grating coupler.

The OFS is realized by applying a serrodyne modulation in a TO phase modulator on the reference arm, which basically includes an optical waveguide, a Titanium heater, and an intermediate layer of Benzocyclobutene (BCB) which is used to avoid strong optical absorptions by the heater. The thickness of the BCB is around $600 \mathrm{~nm}$. By applying a repetitive square-root-of-time voltage across the heater and creating a temperature change in the underlying waveguide, a sawtooth phase change can be generated in the guided light. In order to create a maximal phase shift of $2 \pi$, the peak-to-peak voltage of the driving signal is adjusted and chosen to be $5.62 \mathrm{~V}$, which corresponds to an averaging power is around $16 \mathrm{~mW}$. Due to the bandwidth limit of the heater on $\mathrm{BCB}$, the generated frequency shift is only $2 \mathrm{kHz}$.

The the test setup for the on-chip LDV is schematically shown in figure 2. The light used for the measurement is sent into the chip from an external DFB laser via a fiber. Two aspherical lenses are used to focus the measurement light perpendicularly onto a mirror which is attached to a piezo-stack. The vibration of the piezo-stack is controlled by a sinusoidal voltage source. The mixed light on chip is sent to an external photo-detectors via another fiber. In this measurement, a fiber array was used instead of two fibers.

The flow chart of the demodulation process in the computer is shown in figure 3. With this method, two signals ( $I$ and $Q$ ) with orthogonal phases are generated. An I\&Q vector graph is

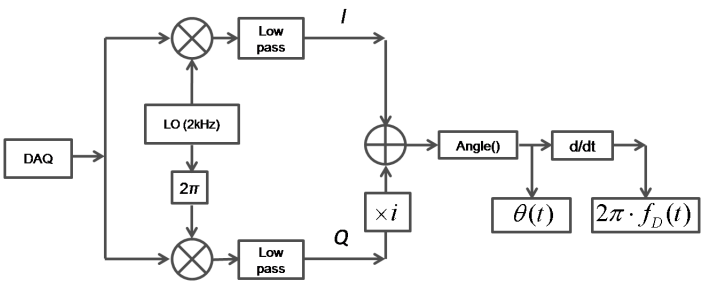

Figure 3. Flow chart for the numerical demodulation.

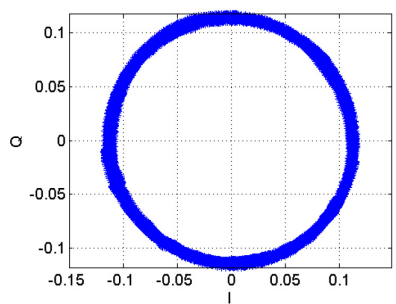

(a)

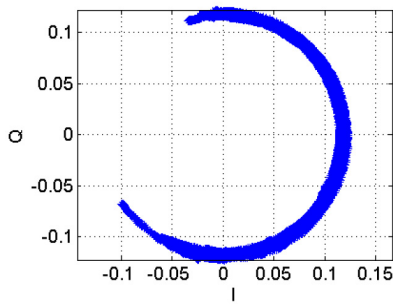

(b)
Figure 4. I\&Q vector trace for two different vibrations: 4(a) is a vibration with the amplitude larger than half a wavelength of the light; 4(b) is one with amplitude less than half a wavelength. Both figures are from results measured by the on-chip LDV.

plotted in figure 4 . The angle of the vector directly relates to the displacement of the mirror. It is sometimes found that the center of the circle is not in the origin. This deviation is mainly caused by the spurious reflections in the chip and a bad driving signal in the serrodyne OFS. The spurious reflections may occur on a grating coupler, a beam splitter, a small waveguide bend or even the backside of the chip. A numerical method was implemented to shift the center of the circle back to the origin. Although this method can somehow compensate the deviations due to the spurious reflections, the noise introduced by these spurious reflections cannot be fully canceled. Many efforts should be done to suppress spurious reflections as much as possible. If the serrodyne signal is not good enough, the circle can also become an ellipse, with its eccentricity determined by the relative strength of the other order harmonics compared with that of the fundamental order. The thickness of the edge of the I\&Q curve is caused by the noise from the laser.

\section{Measurement Results}

Displacement results recovered from the on-chip LDV are compared with those from a commercial LDV from Polytec. The time dependent displacements for both measurements are shown in figure 5. Note that the two measurements were done separately, so we didn't try to match the phases of the results. The curves shown in figure 5(a) are for the measurements on a piezo-stack driven by a $22.6 \mathrm{~Hz}$ sinusoidal voltage signal with a peak-to-peak value of $50 \mathrm{~V}$. The curves in figure $5(\mathrm{~b})$ are for the same vibrating frequency but with a peak-to-peak driving voltage of $100 \mathrm{~V}$. The vibrations were distorted due to the excitation of higher acoustic harmonic orders. It can be seen that in both cases, the vibration shapes recovered from the on-chip LDV are quite similar to those from Polytec. The 


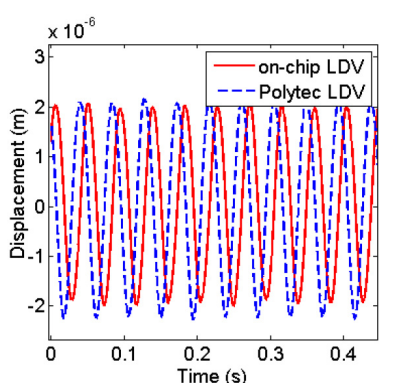

(a)

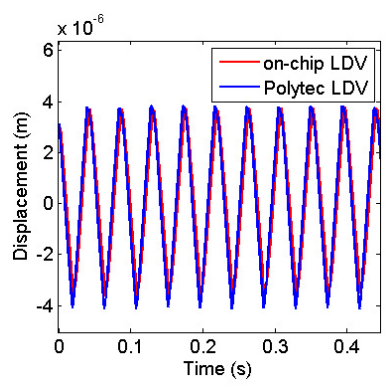

(b)
Figure 5. Demodulated displacements for on-chip LDV: the solid red curve stands for results measured with on-chip LDV, and the blue dashed lines are for results from Polytec LDV. Results in 5(a) are for the piezo vibration driven by a $50 \mathrm{Vpp}$ signal, while those in 5(b) are for the vibration driven by 100 Vpp.

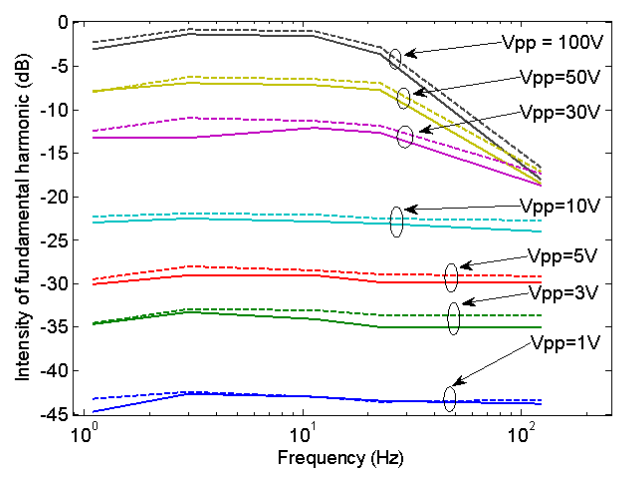

Figure 6. Response of the piezo-electric stack measured both by on-chip LDV (solid lines) and Polytec LDV (dashed lines). The peak-to-peak voltages of the piezo driver are chosen as: $1 \mathrm{~V}, 3 \mathrm{~V}, 5 \mathrm{~V}, 10 \mathrm{~V}, 30 \mathrm{~V}, 50 \mathrm{~V}$, and $100 \mathrm{~V}$.

amplitudes from both results are quite close to each other, but not exactly the same.

The frequency responses of the fundamental harmonic orders of the piezo stack measured by both the on-chip and Polytec LDVs are shown in figure 6. It is shown that the measured responses are quite close to each other. However, the results from the on-chip LDV were usually lower than those from Polytec, and the amplitude ratios are mostly within the region between $90 \%$ and $95 \%$. These differences are still under further investigations.

Due to the speed limit of the thermo-optic based serrodyne OFS, the frequency and amplitude of the vibrations should be limited. In order to measure vibrations with larger frequency or larger amplitudes, a fast optical frequency shifter is needed. This might be realized by using a carrier injection/depletion optical phase modulator, whose bandwidth would be larger than several gigahertz [7]. The maximal vibration velocity shown in this measurement is around $400 \mu \mathrm{m} / \mathrm{s}$, and the minimal velocity is around $4 \mu \mathrm{m} / \mathrm{s}$. It is hard to show the results for the lower limit because a lot of environmental noises are mixed with the useful signal.

\section{CONCLUSION}

We have demonstrated an on-chip laser Doppler vibrometer with a thermo-optic phase modulation based serrodyne optical frequency shifter. Vibrations of a piezo stack within the frequency range $[1.1 \mathrm{~Hz}, 303 \mathrm{~Hz}]$ and the velocity range between [4 $\mu \mathrm{m} / \mathrm{s}, 400 \mu \mathrm{m} / \mathrm{s}$ ] were measured with the on-chip LDV. The measurement results were compared with a commercial LDV from Polytec. It is shown that on-chip LDVs are able to retrieve the instantaneous displacements and velocities within this range.

\section{ACKNOWLEDGEMENT}

The authors acknowledge the Ghent University-Methusalem project "Smart Photonic Chips" for financial supports. The authors thank Xin Yin for useful discussions.

\section{REFERENCES}

[1] H. Toda, M. Haruna, and H. Nishihara, "Optical integrated circuit for a fiber laser doppler velocimeter," Journal of Lightwave Technology, vol. 5, no. 7, pp. 901-905, 1987.

[2] C. I. Moir, "Miniature laser doppler velocimetry systems," OPTICAL SENSORS 2009, vol. 7356, no. L73560I , 2009.

[3] R. G. Brown, J. G. Burnett, J. Mansbridge, C. I. Moir, and B. S. Lowans, "Miniature, solid state photon correlation laser Doppler velocimetry." Applied optics, vol. 29, no. 22, pp. 3291-302, Aug. 1990.

[4] W. Bogaerts, R. Baets, and P. Dumon, "Nanophotonic waveguides in silicon-on-insulator fabricated with CMOS technology," Journal of Lightwave Technology, vol. 23, no. 1, pp. 401-412, 2005.

[5] Y. Li and S. Meersman, and R. Baets "Optical frequency shifter on SOI using thermo-optic serrodyne modulation," 7th IEEE International Conference on Group IV Photonics (GFP), pp. 75-77, 2010.

[6] D. Vermeulen, Y. D. Koninck, Y. Li, E. Lambert, W. Bogaerts, R. Baets, and G. Roelkens "Reflectionless grating coupling for silicon-on-insulator integrated circuits," 8th IEEE International Conference on Group IV Photonics (GFP), pp. 74-76, 2011.

[7] W. Green, M. Rooks, L. Sekaric, and YA. Vlasov "Ultra-compact, low RF power, $10 \mathrm{~Gb} / \mathrm{s}$ silicon MachZehnder modulator," Optics Express, vol. 15, no. 25, pp. 17 106-17 113, 2007. 